\title{
Optimization of shut-in time before drilling for each strata system in the drilling pass area in Block $G$
}

\author{
Lijun Huang ${ }^{1}$ \\ ${ }^{1}$ The 3rd Oil Production Factory, Daqing Oil Field Company Ltd, Daqing, China
}

\begin{abstract}
After the A oilfield enters the ultra-high water cut period, the production level is high, the comprehensive water cut is high and the production level is high. In recent years, the number of new wells has increased considerably, affecting the increase in production year by year. Faced with these two contradictions, a new scientific and effective drilling gateway management model has been explored to reduce the impact of drilling gateways on production. This will have a very important guarantee for the realization of A oilfield's continued stable production. Based on the research and practice of the drill-off area in Block G, this paper determines the minimum injection stop time before drilling-off corresponding to each set of formations according to the bottom hole pressure change law of the injection-stop well.
\end{abstract}

\section{Basic overview of the Block $G$}

Since block $G$ was put into development, the water injection drive well pattern has been divided into four sets of well patterns, which are the basic well pattern for mining the main oil layer of $\mathrm{S}$, and the one-time intensification adjustment well pattern for mining the P II $\mathrm{G}$ medium and low permeability layer. The secondary infilling adjustment well pattern of poor oil layers, the exploitation of S, P, G differential layers and the tertiary infilling well patterns of unused oil layers, the current $P$ basic well pattern has been fully utilized by PI polymer flooding.

\section{Determination of shut-in time before drilling}

A Oilfield proposed the concept of shut-off drilling in 1975, which means that in order to control formation pressure during drilling, ensure drilling safety and improve cementing quality, oil production plants need to shut in some water injection wells to depressurize before and during drilling. Based on past experiences, water injection wells within 450 meters from the new drilling site must be shut in and depressurized 15-20 days before drilling.

However, from the perspective of the development status of the oil layer, the oil layer in the block has strong heterogeneity, there are differences in the plane and the vertical direction, the layer with high permeability and good oil layer conditions, and the pressure drop rate is fast after the water injection well is shut in. Wells with medium and low permeability, good oil layer conditions, average depressurization rate, poorly developed oil layers, and high formation pressure wells have slow depressurization rate. Therefore, it is necessary to optimize the shut-in time before drilling according to the conditions of the well pattern, the development status of the oil layer, and the pressure drop rate of different types of oil layers after shut-in, to ensure the shortest shut-in time, so that the shut-in management from extensive to refined change.

From 2017 to 2020, after three years of research on the shut-in well area in Block $G$, the formation pressure distribution field of the block was analyzed, and the drilling shut-off range of each set of strata was determined. After the injection stop range was determined, different calculations. The formation pressure at the point to be drilled under different stop injection times in the well pattern further determines the stop injection time before drilling.

\subsection{Drilling range of the basic well pattern}

Taking the point to be drilled P0 $(2250,2000)$ as an example, a reasonable drilling range of the point to be drilled is required, that is, to determine the well that controls the formation pressure of the point to be drilled. Taking point $\mathrm{P} 0$ as the center, stop the injection of the water injection wells sequentially from near to outside, and obtain the pressure at the point $\mathrm{P} 0$ to be drilled.

The calculation results of the formation pressure at the point $\mathrm{P} 0$ to be drilled when the injection is stopped for 30 days under different injection stops are shown in Figure 1.

\footnotetext{
${ }^{a}$ Corresponding author: ljhuang@petrochina.com.cn
} 


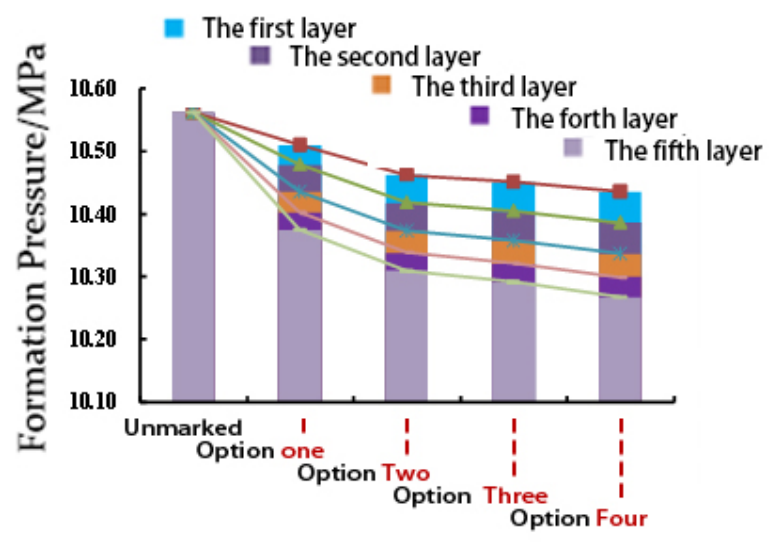

Stop Injection Plan

Figure1.Changes of formation pressure in each sublayer during 30 days of shut-in in different shut-in ranges

When the shut-in range is greater than $450 \mathrm{~m}$, the formation pressure drop decreases significantly with the increase of the shut-in range. That is, the formation pressure at the point to be drilled is more significantly affected by water injection wells within $450 \mathrm{~m}$. Therefore, it is determined that the stop range of P0 to be drilled is $450 \mathrm{~m}$.

\subsection{One-time well pattern drilling range}

In the same way, select the point $\mathrm{P} 0$ to be drilled in an infill well. With P0 as the center, five sets of injection stop programs are designed to stop the injection of the water injection wells from near to the outside, and the pressure at the point $\mathrm{P} 0$ to be drilled is calculated. When the injection is stopped for 30 days under different injection stop schemes, the calculation result of the formation pressure at the point to be drilled P0 is: when the shut-in range is greater than $350 \mathrm{~m}$, the formation pressure drop decreases significantly with the increase of the shut-in range, that is, the formation pressure at the point to be drilled The influence of water injection wells within $350 \mathrm{~m}$ is more significant. Therefore, it is determined that the stop range of the point $\mathrm{P} 0$ to be drilled is $350 \mathrm{~m}$.

\subsection{Secondary well pattern drilling range}

The deployment method of the secondary well pattern is the same as that of the primary well pattern. According to the same method, the formation pressure calculation result of the point $\mathrm{P} 0$ to be drilled when the injection is stopped for 30 days under different injection stop schemes is: when the shut-in range is greater than $350 \mathrm{~m}$, the formation pressure drop decreases significantly with the increase of the shut-in range, that is to say The formation pressure at this point is more significantly affected by water injection wells within $350 \mathrm{~m}$. Therefore, it is determined that the stop range of the point $\mathrm{P} 0$ to be drilled is $350 \mathrm{~m}$.

\subsection{Three-time well pattern drilling range}

The calculation result of the formation pressure at the point $\mathrm{P} 0$ to be drilled when the injection is stopped for 30 days under different injection stop schemes is: when the shut-in range is greater than $350 \mathrm{~m}$, the formation pressure drop significantly decreases with the increase of the shut-in range, that is, the formation pressure at the point to be drilled is affected The impact of water injection wells within $350 \mathrm{~m}$ is more significant. For reservoirs with different permeability, when the shut-in range is greater than $350 \mathrm{~m}$, the greater the permeability of the reservoir, the greater the drop in formation pressure, that is, the greater the impact of the formation pressure at the point to be drilled by the water injection well beyond $350 \mathrm{~m}$. Therefore, it is determined that the stop range of the point $\mathrm{P} 0$ to be drilled is $350 \mathrm{~m}$.

\section{Determination of shut-in time before drilling for each set of strata}

The water injection well in the current drilling block is drilled without overflow. When the measured wellhead pressure is lower than $3 \mathrm{MPa}$, it is considered [1] that the pressure required for drilling is satisfied. When the water injection well is shut down, the wellhead pressure of the water injection well continues to decrease over time, which indirectly reflects that the bottom hole pressure is also constantly decreasing. The bottom hole pressure is composed of the following factors:

$$
p_{w}=p_{c}+p_{l}
$$

formula: Pw—Bottom hole pressure $\mathrm{MPa}$;

Pc_-Wellhead pressure, $\mathrm{MPa}$;

Pl_Annular liquid column pressure, MPa.

Due to the non-discharge method, the wellbore can be considered full. Therefore, the value of Pl will not change basically, and the change trend of bottom hole 
pressure $\mathrm{Pw}$ basically reflects the change trend of wellhead pressure.

Under the condition that all water injection wells of each set of strata within the range of injection stop are stopped, the bottom hole pressure changes with the stop injection time are as follows (Figure 2, Figure 3, Figure 4, Figure 5).

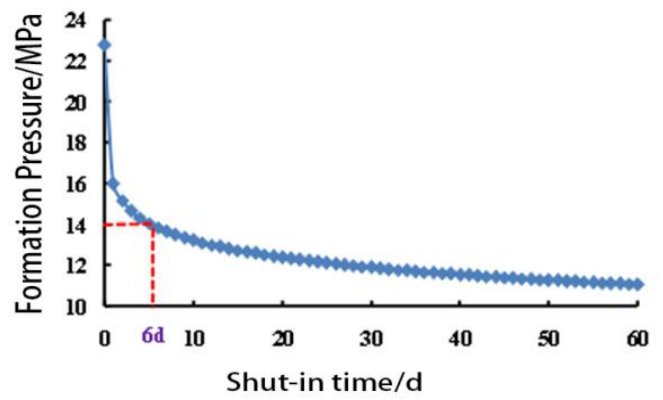

Figure2. The pressure of changes in basic wells with time of stopping injection

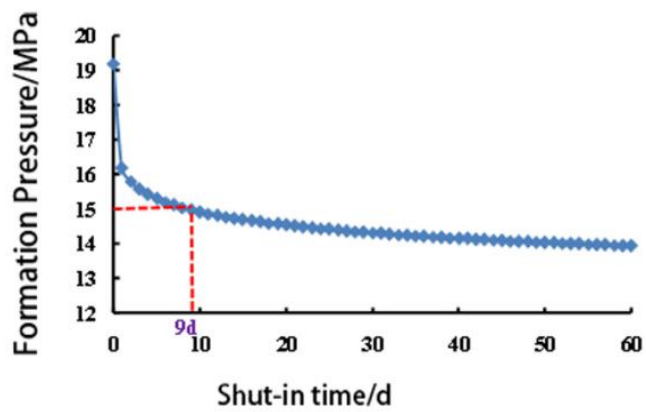

Figure3. The pressure of changes in one-time dense wells with time of stopping injection

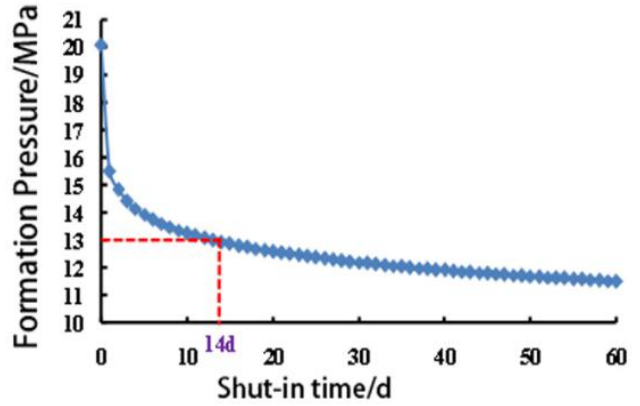

Figure4. The pressure of changes in two-time dense wells with time of stopping injection

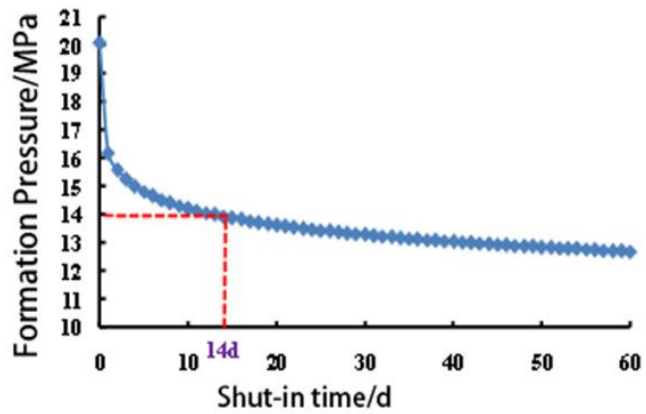

Figure5. The pressure of changes in three-time dense wells with time of stopping injection

In the drill-off practice of Block $\mathrm{G}$, the drill-off area realized that when the wellhead pressure of the injection well whose injection was stopped is less than or equal to $3 \mathrm{MPa}$, the injection-stop time can be considered as the drill-off requirement. Based on this, we determined the minimum injection suspension time before drilling of the corresponding basic well pattern to be $6 \mathrm{~d}$, the minimum injection suspension time for the primary pattern was $9 \mathrm{~d}$, 
and the minimum injection suspension time before the secondary pattern drilling It is $14 \mathrm{~d}$, and the minimum injection stop time before three well pattern drilling is $14 d$.

\section{Evaluate the effect of field application}

While applying research results to rationally optimize the shut-in radius, considering that there are sufficient pressure relief points at the edge of the drilling-in area, the shut-in distance at the edge of the drilling-in area is optimized from $450 \mathrm{~m}$ to $300 \mathrm{~m}$, which effectively reduces the number of drilling-in wells. In the past three years, the planned average shut-in time is 53.3 days, and the actual shut-in time is 31.7 days, which is 21.6 days less than planned and 39 days shorter than before optimization. Drilling shut-in affects production.

\section{Conclusion}

5.1 The reasonable drilling range of well patterns in different layers is determined. The pre-drilling stop time can be determined by calculating the formation pressure at the point to be drilled under different stop injection times and the bottom hole pressure change of the water injection well within the stop injection range.

5.2 By studying the dynamic change characteristics of the drill-off area, according to different regions and different geological conditions, different strata nets adopt different pre-drilling shut-in times, which is very necessary to reduce the impact of drilling off on production.

\section{References}

1. Zhou L W, Oil and gas field surface engineering. An effective way to optimize drilling methods. 8:35-37. (2004)

2. Xu Baocheng, Xu Baolong. A brief discussion of the impact of drilling and the understanding of the law. Heilongjiang Science and Technology Information.7: 24 (2010)

3. Liu Ding zeng, Wang Qimin, Li Bohu. The seepage ch aracteristics of the reservoir. Daqing multi - layer sa ndstone oil field development.9: 50-58 (2006) 